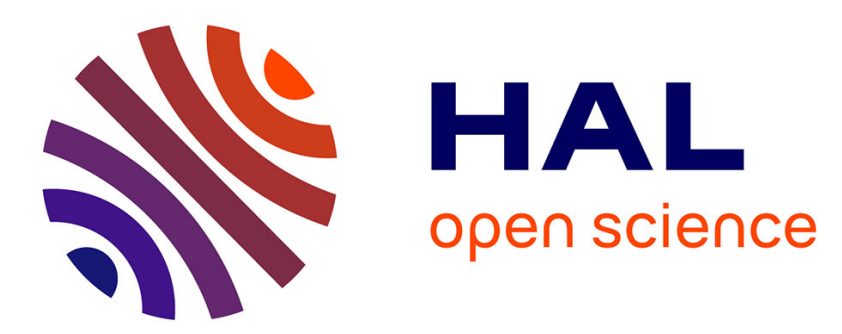

\title{
Importance of patient DTI's to accurately model glioma growth using the reaction diffusion equation
}

Erin Stretton, Ezequiel Geremia, Bjoern H. Menze, Hervé Delingette, Nicholas Ayache

\section{- To cite this version:}

Erin Stretton, Ezequiel Geremia, Bjoern H. Menze, Hervé Delingette, Nicholas Ayache. Importance of patient DTI's to accurately model glioma growth using the reaction diffusion equation. 2013 IEEE International Symposium on Biomedical Imaging: From Nano to Macro, 2013, San Francisco, CA, United States. pp.1130-32. hal-00840036

\section{HAL Id: hal-00840036 https://hal.inria.fr/hal-00840036}

Submitted on 1 Jul 2013

HAL is a multi-disciplinary open access archive for the deposit and dissemination of scientific research documents, whether they are published or not. The documents may come from teaching and research institutions in France or abroad, or from public or private research centers.
L'archive ouverte pluridisciplinaire HAL, est destinée au dépôt et à la diffusion de documents scientifiques de niveau recherche, publiés ou non, émanant des établissements d'enseignement et de recherche français ou étrangers, des laboratoires publics ou privés. 


\title{
IMPORTANCE OF PATIENT DTI'S TO ACCURATELY MODEL GLIOMA GROWTH USING THE REACTION DIFFUSION EQUATION
}

\author{
E. Stretton $\quad$ E. Geremia $\quad$ B. Menze $^{\star} \quad$ H. Delingette $\quad$ N. Ayache . $^{\star}$ \\ * Asclepios Research Project, INRIA Sophia Antipolis Mediterranee, ${ }^{\dagger}$ ETH Zurich
}

\begin{abstract}
Tumor growth models based on the FisherKolmogorov reactiondiffusion equation (FK) have shown convincing results in reproducing and predicting the invasion patterns of gliomas brain tumors. Diffusion tensor images (DTIs) were suggested to model the anisotropic diffusion of tumor cells in the brain white matter. However, clinical patient-DTIs are expensive and often acquired with low resolution, which compromises the accuracy of the tumor growth models. In this work, we used the traveling wave approximation model to describe the evolution of the visible boundary of the tumor modeled by the FK equation to investigate the impact of replacing the patient DTI by (i) an isotropic diffusion map or (ii) an anisotropic high-resolution DTI atlas formed by averaging DTIs of multiple patients. We quantify the impact of replacing the patient DTI using three metrics: the shape of the simulated glioma, the estimation of the tumor growth parameters, and the prediction performance on clinical cases.
\end{abstract}

Index Terms - reaction-diffusion equation, DTI, glioma

\section{INTRODUCTION}

Gliomas are a type of malignant brain tumor with fast infiltration along white matter fiber tracts. Modeling glioma growth is a complex task due to the infiltrative nature of this disease, which makes low concentrations of tumors unobservable in magnetic resonance images (MRIs). Several biophysical computational models have been proposed for describing the diffusive growth of these tumors that cannot be completely seen in MRIs. Swanson et al. [1] proposed the use of tissue-based tumor cell motility, where their model included different diffusivities in white and grey matter, but isotropic and homogeneous within each. To improve the realism of the model, Clatz et al. [2], Jbabdi et al. [3] and Menze et al. [4] constructed anisotropic non-homogeneous models within white matter based on patients DTIs. Unfortunately, patient DTIs are not widely available because of the added cost and time for acquisition. Clinical patient DTIs are usually of low quality (low-resolution and low signal-to-noise ratio) due to the limited number of diffusion gradients and repetitions acquired. Although there exist several sequences to

Care4ME ITEA2-ip08004 \& ERC Adv. Grant MedYMA 2011-291080 accelerate DTI acquisitions (e.g., SENSE), they are not systematically used in a clinical setting. In addition, the white matter tracts on the patient DTIs are often disturbed inside the region of the tumor and close to the boundary causing the image to be incomplete. It is for this reason that Unkelbach et al. [5] proposed to use Swanson's model without a DTI for radiotherapy planning. Alternatively, instead of relying on a patient-specific DTI ("patient-DTI"), one may rely on a DTI atlas ("atlas-DTI," which is an average of 75 subject DTIs [6]), or refrain from using a DTI at all ("no-DTI"). To date, there has been no analysis carried out to quantify whether the patient specific information on anisotropic tumor growth directions in patient-DTI gives invaluable information in terms of tumor growth prediction.

In this work we provide a comparison between three modeling options: using a patient-DTI, atlas-DTI and no-DTI. We compare these three options and evaluate which method aids the traveling wave approximation model in providing the most accurate description of the tumor growth dynamics. This study also gives insight into whether the tumor growth has directional preference (anisotropic growth) as formulated in $[2,3]$ or only obeys tissue based differential motility as proposed in [1]. This question is crucial since most of the available data do not have DTIs. We compare these modeling options using three different metrics: the shape of the simulated glioma, the estimation of the tumor growth parameters, and the prediction performance on a few clinical cases.

\section{TENSOR FORMS FOR THREE DTI OPTIONS}

Consider the Fisher-Kolmogorov to describe the 3-D macroscopic dynamics of the tumor cell density $u$ :

$$
\frac{\partial u}{\partial t}=\underbrace{\nabla \cdot(D(\mathbf{x}) \nabla u)}_{\text {Diffusion Term }}+\underbrace{\rho u(1-u)}_{\text {Logistic Reaction Term }} ; \underbrace{\eta_{\partial \omega} \cdot(D \nabla u)=0}_{\text {Boundary Condition }}
$$

where the diffusion tensor $D(\mathbf{x})$ depends on the position $\mathbf{x}, \rho$ is the proliferation rate, $\eta_{\partial \omega}$ are the normal directions to the boundaries of the brain surface, and $t$ represents time.

Different $D(\mathbf{x})$ constructions have been proposed in the past. Some researchers $[1,5,2]$ have considered isotropic diffusivity and defined $D(\mathbf{x})$ as $d_{g} I$ if $\mathbf{x}$ is in gray matter 


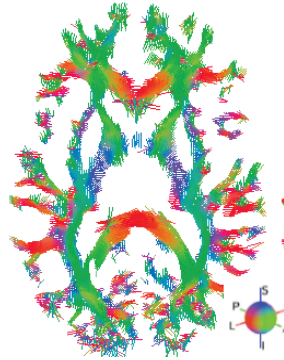

(a) Subject-DTI

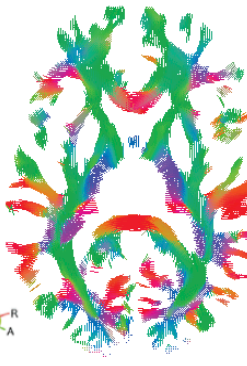

(b) Atlas-DTI

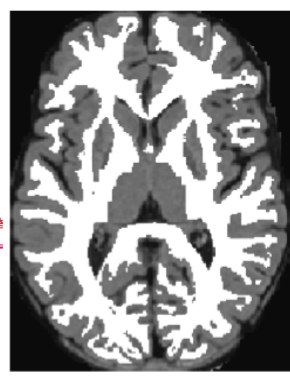

(c) WM Seg on FLAIR
Fig. 1. Diffusion tensor forms. (a) \& (b) show water diffusion tensor directions that were used to model anisotropic growth in WM. (c) WM segmentation that was used to model region where tumor would isotropically grow for the no-DTI option.

and $d_{w} I$ if $\mathbf{x}$ is in white matter, where $d g$ and $d w$ are scalars $(d g<<d w)$ and $I$ is the identity matrix. This representation is used in this paper for the no-DTI case, where the isotropic and homogeneous growth in white matter (WM) is guided by a WM segmentation (Figure 1(c)). Other researchers [2, 4, 7, 8] have examined anisotropic diffusivity where $D(\mathbf{x})$ is $d_{g} I$ if $\mathbf{x}$ is in gray matter and $d_{w} D_{\text {water }}$ if $\mathbf{x}$ is in white matter, where $D_{\text {water }}$ is the water diffusivity tensor obtained from a DTI normalized using the maximum eigenvalue in white matter [2]. These $D_{\text {water }}$ based DTIs are used for the patient-DTI and atlas-DTI cases. Even though patient-DTIs represent better the patient's white matter architecture, they also have the drawbacks of having less resolution and more noise. On the other hand, atlas-DTIs are less noisy as a result of the averaging caused by using multiple patients [6]. Figure 1 shows a comparison between a patient-DTI (a) and an atlas-DTI (b). To the best of our knowledge, there is no study that gives a clear indication of which $D(\mathbf{x})$ construction can be used in a FK model to produce the best tumor growth prediction results measurable by FLAIR MRIs.

To overcome the fact that cell density $u$ in Equation (1) cannot be directly observed, we use the traveling wave formulation [7] to approximate the evolution of the tumor boundary that can be observed in the MRIs. The model's inputs are two tumor segmentations from different dates (delineated from FLAIR MRIs), brain and white matter segmentations (found from a T1 MRI), and a tensor image (one of the DTI options) to describe the tumors diffusion in white matter. The model must first be personalized to find patient optimum modeling parameters (i.e., tumor growth speed that is a function of: $d w * \rho$ in white matter and $d g * \rho$ in gray matter). The model's output is the predicted tumor evolution in isotime contours.

\section{SHAPE OF THE SIMULATED GLIOMA}

How close are the simulation results using an atlas-DTI or no-DTI to the results using the subject-DTI?

A synthetic dataset was formed by a healthy subject-DTI, a T1 MRI, a FLAIR MRI, and an atlas-DTI. We registered

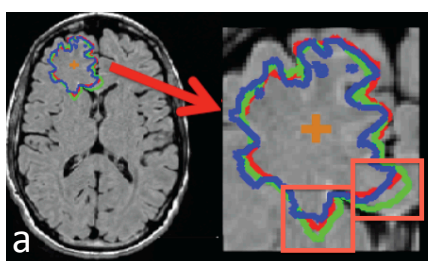

LGG in the frontal lobe

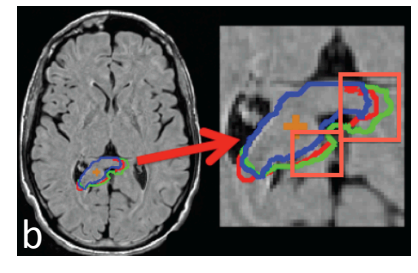

HGG in the corpus callosum
Fig. 2. High-resolution synthetically grown tumors. AtlasDTI (green) and no-DTI (blue) contours are compared to subject-DTI (red). Start seed is in orange.

all images to the Apparent Diffusion Coefficient map (ADC) of the subject-DTI (rigid for the subject-T1 and non-rigid for the atlas-DTI that was taken from [6]) for the purpose of being able to compare results.

We compared similar volumes of the atlas and no-DTI options to the subject-DTI using the root-mean-squared symmetric distance error (RMSE) metric where we compared the isosurfaces (i.e., simulation results with segmentation at a give time point). Using the approach described in Section 2, 120 synthetically grown tumor evolutions were initialized from a single voxel at randomly chosen locations. The simulation results from using the subject-DTI were used as the comparison baselines (i.e., thresholded at 200 days of tumor growth). The speed related to the three $D(\mathbf{x})$ s were not directly comparable since the atlas and subject-DTIs were normalized differently and DTIs modeled non-homogeneous diffusion in white mater. Furthermore, the $D(\mathbf{x})$, which was not based on DTIs (no-DTI option) was used to model homogeneous tumor diffusion in white matter. Therefore, to provide a meaningful comparison, tumor growths were stopped when the tumor volume (for the atlas-DTI and no-DTI options) were the same as the ones obtained after 200 days with the subject-DTI. The synthetic experiments included comparing the RMSE found using the atlas and no-DTI options when the MRIs had different resolutions (low-resolution case $=1 \mathrm{~mm} \times 1 \mathrm{~mm} \times 3 \mathrm{~mm}$, high-resolution case $=0.5 \mathrm{~mm} \times$ $0.5 \mathrm{~mm} \times 1.5 \mathrm{~mm}$ ), tumor locations (Supratentorial and Infratentorial regions), and various tumor grades (high grade glioma [HGG]: $d w=0.5 \mathrm{~mm}^{2} / \mathrm{day}, \mathrm{dg}=0.25 \mathrm{~mm}^{2} / \mathrm{day}$, $\rho=0.009 /$ day [units remain the same throughout the article], low grade glioma [LGG]: $d w=0.25, d g=0.01$, $\rho=0.012$, very LGG: $d w=0.1, d g=0.005, \rho=0.024$ ).

The experiments using high-resolution images exhibited a noticeable difference between the results when using the 3 DTI choices and the coarse MRI resolution experiment results did not indicate any visible difference $(<<1 \%$ difference in contour deviation ). Therefore, when using a low-resolution images, it does not matter which type of DTI option is used.

There was an observed difference between the HGG parameter synthetically grown tumors and the slower growing parameter tumors. The tumors developed using HGG parameters had on average a $14 \%$ (supratentorial) and $8 \%$ (infraentorial) difference in contour deviation between no-DTI and 

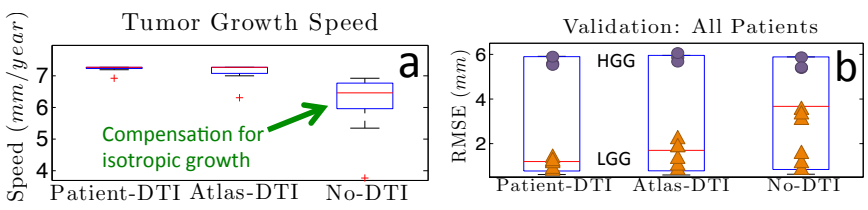

Fig. 3. (a) The no-DTI option grows isotropically, its estimated growth speed must be slower. Depicted is the patient case with 5 ACQs. (b) Validations were performed on the 1st 3 ACQs of all patients showing that HGG results had the most RMSE and the results of the no-DTI option had median (red bar) RMSE higher than the other options.

atlas-DTI to the patient-DTI with respect to total radial tumor growth. The differences were less than $5 \%$ for slower growing tumors. Therefore, when gliomas have a low growth speed $(<40 \mathrm{~mm} / \mathrm{yr})$, it is not as critical which DTI choice is used. This is expected because HGG typically exhibit the anisotropic growth.

Figure 2 shows 2 synthetic tumor cases. The shape of the synthetic tumors look visibly similar for the results of the atlas-DTI (green) and subject-DTI (red) cases due to the fact that both simulate anisotropic tumor growth in white matter where the speed of growth in each area is dictated by the nonhomogeneity of the DTI. As expected, the directional nonhomogeneity of the DTIs is slightly different between the subject and atlas-DTIs in very anisotropic regions (Figure 2 red boxes). The no-DTI (blue) option's tumor growth is homogeneous and isotropic in white matter and therefore does not have an anisotropic growth pattern in white matter, but we still see the change of growth speed between white matter and gray. Despite these differences, shapes of resulting contours from using atlas-DTI and no-DTI options were on average $1 \mathrm{~mm}$ RMSE different than from subject-DTI results.

\section{ESTIMATION: TUMOR GROWTH PARAMETERS}

Can we accurately estimate tumor growth parameters when using each of the three DTI options within the FK model?

We used three clinical data sets with tumors located in the supratentorial region. They were registered the same way as the synthetic-data of Section 3, with the exception that masks were used for the non-rigid registrations to hide the tumors. The tumors were manually segmented in the original FLAIR MRI resolutions and then were registered to the ADC of the patient-DTI. Two of the patients (LGG with low-resolution MRIs, HGG with high-resolution MRIs) had 3 MRI acquisition times and one had 5 acquisitions (LGG with high-resolution MRIs).

Two steps were performed to estimate the tumor growth parameters and analyze how well the 3 DTI options worked within the FK model to reproduce the patient tumor evolutions. (i) Personalization: Optimization of the FK parameters to find the tumor growth speeds (i.e., $d w * \rho, d g * \rho$ ). (ii) Validation: Verification that the simulated tumor contour of

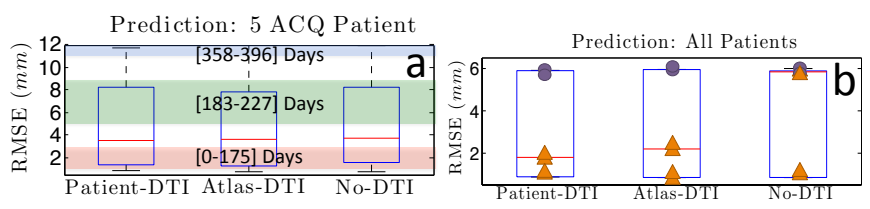

Fig. 4. (a) Model accuracy boundaries, where color corresponds to min and max of error, displayed for the LGG patient with 5 ACQs, show the farther in time one tries to predict, the less accurate the prediction will be. (b) Results are the same as Figure 3(b), with the exception that one of the LGG cases had each of its ACQ times separated by a year with a similar growth rate to the patient depicted in (a), and therefore with isotropic growth had much higher median error (red bar).

the personalization was close in reproducing the actual tumor profile (e.g., Personalize $\left(t_{1}, t_{2}\right)$ Validate $\left.\left(t_{1}, t_{2}\right)\right)$. RMSE was the error metric used. For each of the 3 DTI options, we did 16 experiments ( 10 validations for the patient with 5 acquisitions (ACQ) and 3 for each of the 2 patients with 3 ACQs).

Results showed (i) differences in the estimated tumor growth speed and when validating the growth speed, accuracy differences can be looked at by (ii) grade and (iii) model limitations. First, the no-DTI option estimated tumor growth speed usually has to be slower than the other DTI options since the no-DTI tensor construction method cannot model directional preference and grows isotropically. Therefore, the growth speed needs to compensate. Figure 3(a) shows this for the patient with 5 ACQs. Second, the validation result for all of the patients (Figure 3(b)) shows that HGG had a higher RMSE than LGG had. Third, only the no-DTI case results had a median high RMSE (Figure 3(b)). This was because of model limitations since there was one and two years between ACQs for one of the patients, and the no-DTI case grew too large in the amount of time. This result can also be seen in Figure 4(b) in the prediction experiments.

Despite these differences, the means of the RMSE of the validation experiments for all the patients combined can be considered equal between the patient-DTI, atlas-DTI and noDTI options (p-value $=0.8757$ [patient-DTI vs. atlas-DTI], 0.4626 [patient-DTI vs. no-DTI], 0.5589 [atlas-DTI vs. noDTI], 2 sample t-test).

\section{PREDICTION PERFORMANCE: CLINICAL DATA}

How accurate is the FK model, when using each of the three DTI options, in predicting patient tumor evolutions?

Using the same data from Section 4, we varied the window of estimation and predicted on unseen cases (e.g., Personalize $\left(t_{1}, t_{2}\right)$ Predict(start simulation: $t_{1}$, prediction: $\left.t_{3}\right)$ ). For each of the 3 DTI options, we did 96 experiments $(90$ predictions for the patient with 5 ACQs and 3 for each of the 2 patients with 3 ACQs).

Prediction results show accuracy differences with respect to (i) tumor grade and (ii) model boundaries. First, the pre- 


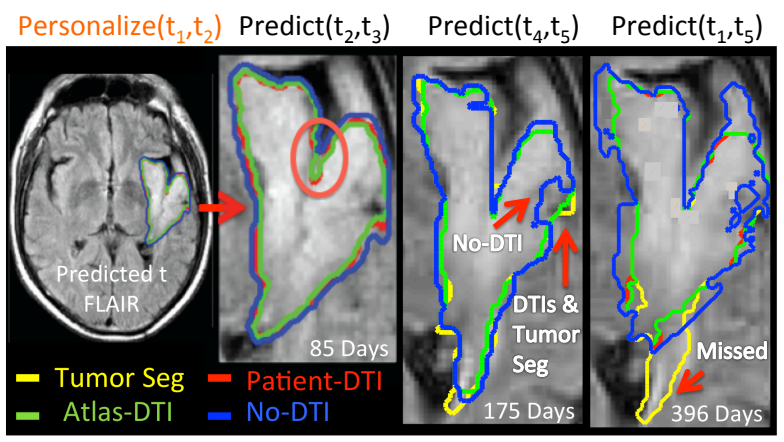

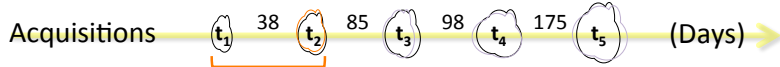

Fig. 5. Results for patient with 5 AQC times.

diction result for all of the patients combined (Figure 4(b)) showed that the HGG had a high RMSE. Second, only the low-resolution no-DTI case results had high RMSE (Figure 4(b)). The low-resolution cases using a DTI had low RMSE. Third, we observed the boundaries of the traveling wave approximation's accuracy with the experiments on the patient with 5 ACQs, where the average tumor growth speed is $6.8 \mathrm{~mm} /$ year (Figure 3(a), 4(a)). Figure 5, shows that for under 175 days, the predicted patient and atlas-DTI case results are practically identical to the actual tumor segmentation (contour lines are sitting on top of each other) and the no-DTI case results are not far off. However, when predicting 396 days out, the no-DTI case results had a lower RMSE than the patient and atlas-DTI options. This is because the tumor was spiky and the results of the results of the no-DTI option grew to be much larger than the results of the patient and atlas-DTI options. Therefore, it would encompass a large region even though it did not match very well the actual tumor segmentation. The patient and atlas-DTI options result contours match much better, even for larger times, the contour of the actual tumor segmentation. However, for large times these DTI options might fail to grow along a spiky white matter path. For example, the DTI tensors for the fingery tumor extension in Day 396 were facing in the right direction for this growth, but the tensor values were low for both the atlas and patient-DTIs, which explains why the model did not choose that path.

\section{DISCUSSION AND CONCLUSIONS}

Three options to model tumor diffusion in white matter within the FK were compared. It was found that the traveling wave approximation model is most accurate for each of the three DTI options when the tumor growth speed is low, the image resolution is high, and the number of days being predicted is low in comparison with the growth speed. The resulted contours of the atlas- and patient-DTI matched the actual tumor contours better than the no-DTI option. However, the results of the no-DTI option using high-resolution images and not too many days with respect to the tumor growth speed were also close to the actual tumor. Therefore, this paper demonstrates that a patient-DTI (when available) is the best option to model tumor cell diffusion in white matter within the FK framework since the results show that tumor growth has directional preference (anisotropic growth) as formulated in [2, 3]. It was also found that not very much accuracy is lost with the use of an atlas-DTI. This study shows that modeling glioma growth with tissue based differential motility (using the no-DTI option) as proposed in [1] yields less accurate results. However, refraining from using a DTI would be fine in situations where the data are very coarse (low resolution), which is the case for many clinics today, if one has a very good white matter mask in the areas surrounding the tumor, or for LGG cases since they are known to grow somewhat isotropically.

\section{REFERENCES}

[1] K.R. Swanson, R.C. Rostomily, and E.C. Alvord, "A mathematical modelling tool for predicting survival of individual patients following resection of glioblastoma: a proof of principle," Br J Cancer, vol. 98, no. 1, 2007.

[2] O. Clatz, M. Sermesant, P.Y. Bondiau, H. Delingette, S.K. Warfield, G. Malandain, and N. Ayache, "Realistic simulation of the 3-d growth of brain tumors in MR images coupling diffusion with biomechanical deformation," IEEE Trans. Med Imag, vol. 24, no. 10, 2005.

[3] S. Jbabdi, E. Mandonnet, H. Duffau, L. Capelle, K.R. Swanson, M. Pélégrini-Issac, R. Guillevin, and H. Benali, "Simulation of anisotropic growth of low-grade gliomas using diffusion tensor imaging," MRM, vol. 54, 2005.

[4] B. H. Menze, K. Van Leemput, A. Honkela, E. Konukoglu, M.A. Weber, N. Ayache, and P. Golland, "A generative approach for image-based modeling of tumor growth," in IPMI. Springer, 2011, pp. 735-747.

[5] J. Unkelbach, B.H. Menze, A. Motamedi, F. Dittmann, E. Konukoglu, N. Ayache, and H. Shih, "Glioblastoma growth modeling for radiotherapy target delineation.," in IGRT MICCAI Workshop, Nice, October 2012.

[6] M. Prastawa, E. Bullitt, and G. Gerig, "Simulation of brain tumors in MR images for evaluation of segmentation efficacy," Medical image analysis, vol. 13, no. 2, 2009.

[7] E. Konukoglu, O. Clatz, B.H. Menze, B. Stieltjes, M.A. Weber, E. Mandonnet, H. Delingette, and N. Ayache, "Image guided personalization of reaction-diffusion type tumor growth models using modified anisotropic eikonal equations," IEEE Med, vol. 29, no. 1, pp. 77-95, 2010.

[8] E. Stretton, E. Mandonnet, E. Geremia, B. H. Menze, H. Delingette, and N. Ayache, "Predicting the location of glioma recurrence after a resection surgery," in STIA MICCAI Workshop. 2012, Springer. 